\title{
ERRATUM
}

To the paper

\section{USING NEURAL NETWORKS TO STUDY NETWORKS OF SCIENTIFIC JOURNALS}

\author{
J. M. CAMPANARIO
}

\section{Grupo de Investigación en Aprendizaje de las Ciencias, Departamento de Física,} Universidad de Alcalá, 28871 Alcalá de Henares Madrid (Spain)

appeared in

Scientometrics, Vol. 33, No. 1 (1995) 23-40.

The followings should be corrected:

1. Page 28: Formula should read

$$
W_{\mathrm{r}}(t+1)=W_{\mathrm{r}}(\mathrm{t})+l_{\text {rate }}(t) \times h_{\mathrm{rs}}(t) \times d\left[X, W_{\mathrm{r}}(t)\right]
$$

2. Page 29: Third paragraph ("as noted above..."). Line number 6: it reads "to 1985 (data set III)". It should read "to 1985 (data set II)".

3. Page 32, last paragraph, line number 4: It reads "mcg, $f,(k) \rightarrow$ "ln" it should read "m, g, f(k) $\rightarrow$ "ln"

4. Pages 33, 34, 35, 36 and 37. Figures are interchanged. Thus map in Figure 3 should be placed in Figure 4 and map in Figure 4 should be placed in Figure 3. Please move only the maps, not the headings of Figures. Map in Figure 5 should be placed in Figure 6 and viceversa. Map in Figure 7 should be placed in Figure 8 and viceversa. Map in Figure 9 should be placed in Figure 10 and viceversa. 